\title{
Effects of PTCs on nonsense-mediated mRNA decay are dependent on PTC location
}

\author{
HEEGYUM MOON* ${ }^{*}$ XUEXIU ZHENG* ${ }^{*}$ TIING JEN LOH, HA NA JANG, \\ YONGCHAO LIU, DA-WOON JUNG, DARREN R. WILLIAMS and HAIHONG SHEN
}

\author{
School of Life Sciences, Gwangju Institute of Science and Technology, Gwangju 61005, Republic of Korea
}

Received August 6, 2015; Accepted October 26, 2016

DOI: $10.3892 / 01.2017 .5627$

\begin{abstract}
The récepteur d'origine nantais (RON) gene is a proto-oncogene that is responsible for encoding the human macrophage-stimulating protein (MSP) 1 receptor. MSP activation induces RON-mediated cell dissociation, migration and matrix invasion. Isoforms of RON that exclude exons 5 and 6 encode the RON $\Delta 160$ protein, which promotes cell transformation in vitro and tumor metastasis in vivo. Premature termination codons (PTCs) in exons activate the nonsense-mediated mRNA decay (NMD) signaling pathway. The present study demonstrated that PTCs at various locations in the alternative exons 5 and 6 could induce NMD of the majority of the spliced, or partially spliced, isoforms. However, the isoforms that excluded exon 6 or exons 5 and 6 were markedly increased when produced from mutated minigenes with inserted PTCs. Furthermore, the unspliced isoform of intron 5 was not observed to be decreased by the presence of PTCs. Notably, these effects may be dependent on the location of the PTCs. The current study demonstrated a novel mechanism underlying the regulation of NMD in alternative splicing.
\end{abstract}

\section{Introduction}

Récepteur d'origine nantais (RON) is a receptor tyrosine kinase and a member of the hepatocyte growth factor receptor proto-oncogene family (1). The RON gene encodes the macrophage-stimulating protein (MSP) 1 receptor (1). The activation of RON by MSP induces tumor progression and invasion, whereas the inactivation of RON promotes cell apoptosis (2-4). $\mathrm{RON}$ is a heterodimeric protein with a disulfide bond linking a $40-\mathrm{kDa} \alpha$ chain, containing the extracellular domain for ligand

Correspondence to: $\mathrm{Dr}$ Haihong Shen, School of Life Sciences, Gwangju Institute of Science and Technology, 123 Cheomdangwagi-ro, Buk-gu, Gwangju 61005, Republic of Korea E-mail: haihongshen@gist.ac.kr

${ }^{*}$ Contributed equally

Key words: récepteur d'origine nantais proto-oncogene, pre-mRNA splicing, stop-codon, nonsense-mediated mRNA decay binding, and a $150-\mathrm{kDa} \beta$ chain, incorporating the intracellular kinase domain and a transmembrane domain $(5,6)$. The binding of MSP to RON activates the intrinsic kinase activity and induces autophosphorylation of the tyrosine residues in its kinase domain (7). Alternative splicing of the RON pre-mRNA produces various proteins, each with distinctive functions (8). RON $\Delta 165$, which is produced by the exclusion of exon 11, has previously been observed to accumulate in breast and colon cancer cells, where it acts to stimulate invasive cell growth and metastasis $(9,10)$. Previous studies have demonstrated that serine/arginine-rich splicing factor (SRSF) 1 and heterogeneous nuclear ribonucleoprotein A1 regulate the epithelial-to-mesenchymal transition through the regulation of exon 11 alternative splicing in RON pre-mRNA $(10,11)$. Our previous study also identified SRSF2 as an important regulator protein for the alternative splicing of exon 11 (12). Another isoform, RON $\Delta 160$, is produced by the exclusion of exons 5 and 6 and lacks the first immunoglobulin-plexin-transcription domain in the extracellular $\beta$ chain, and therefore induces cellular transformation, metastasis and protects tumor cells from apoptosis (13-15). The mechanisms underlying the alternative splicing of exon 5 and 6 require further exploration.

Pre-mRNA splicing is required for gene expression in higher eukaryotes, as introns must be removed from pre-mRNA and protein-coding exons must be ligated (16). mRNA splicing is a two-step procedure. Initially, the 5' splice site is cleaved and two splicing intermediates are generated, a linear first exon and an intron-second exon RNA species in a lariat configuration (17); a 2'-5' phosphodiester bond is formed between the guanine at the 5 ' splice site and the 2 ' hydroxyl of an adenine at a branch point (18). Secondly, the 3 ' splice site is cleaved and the two exons are ligated together to generate the spliced mRNA (19). Nonsense-mediated mRNA decay (NMD) is a method of eliminating aberrant mRNA transcripts that contain premature termination codons (PTCs) (20). If PTCs are included in mRNA, or partially spliced mRNA, through alternative splicing, then NMD may occur (21). NMD has a role in the quantitative posttranslational regulation of gene expression through specific alternative splicing $(22,23)$; however, the underlying regulatory mechanisms require further studies to be fully elucidated.

The results indicated that PTCs at specific locations affect the splicing and NMD of various isoforms to alternate degrees. These results may be applicable in treating certain types of 
cancer, decreasing cancer-specific or metastasis-specific RNA isoforms via inducing PTCs using gene editing.

\section{Materials and methods}

Plasmid construction. RON exon 4-7 sequences were amplified from human genomic DNA using E4Bam.F and E7Xho.R primers (Table I); the products were cloned into a pcDNA3.1 (+) vector at $B a m \mathrm{HI}$ and $\mathrm{XhoI}$ cleavage sites to produce a minigene. E5/SC-1, E5/SC-2, E6-7/SC-1 and E6-7/SC-2 constructs were made using overlapping polymerase chain reaction (PCR); the aforementioned minigene provided the template, and following primers were used: E5-M1.F/E5-M1.R for the E5/SC-1 minigene, E5-M2.F/E5-M2.R for the E5/SC-2 minigene, E6Tins.F/E6Tins.R and E7Gdel.F/E7Gdel.R for the E6-7/SC-1 minigene, and E6Ains.F/E6Ains.R and E7Tdel.F/E7Tdel.R for the E6-7/SC-2 minigene. The PCR cycling settings were as follows: $5 \mathrm{~min}$ at $95^{\circ} \mathrm{C}$, followed by 30 cycles of denaturation for $15 \mathrm{sec}$ at $95^{\circ} \mathrm{C}$ and annealing for $1 \mathrm{~min}$ at $60^{\circ} \mathrm{C}$. A 0.5 unit Taq DNA polymerase (Cosmo Genetech Co., Ltd., Seoul, Korea) and 0.2 mM dNTPs (Cosmo Genetech Co., Ltd.) were used in the PCR reaction. All of the primer sequences are listed in Table I.

Cell culture and transfection. MDA MB 231 cells (ATCC, Manassas, VA, USA) were grown in RPMI 1640 medium (HyClone; GE Healthcare Life Sciences, Logan, UT, USA) supplemented with $10 \%$ fetal bovine serum (FBS) (HyClone; GE Healthcare Life Sciences) at $37^{\circ} \mathrm{C}$ with $5 \% \mathrm{CO}_{2}$. Polyethylenimine (PEI; Sigma-Aldrich; Merck Millipore, Darmstadt, Germany) was used to transfect the minigenes into the cells.

Reverse transcription (RT)-PCR. A RiboEx reagent (GeneAll Biotechnology Co., Ltd., Seoul, Korea) was used to extract the total RNA from the cells. A total of 40 units ImProm-II ${ }^{\mathrm{TM}}$ reverse transcriptase (Promega Corporation, Madison, WI, USA) was used to reverse transcribe $1 \mu \mathrm{g}$ RNA with 100 pmol oligo dT and $0.2 \mathrm{mM}$ each dNTP in $20 \mu \mathrm{l}$ reaction. The RT reaction product $(1 \mu \mathrm{l})$ was amplified by PCR using G-Taq polymerase (Cosmo Genetech Co., Ltd.) and the following PCR settings: Denaturing for $5 \mathrm{~min}$ at $95^{\circ} \mathrm{C}$, followed by 32 cycles of denaturation for $15 \mathrm{sec}$ at $95^{\circ} \mathrm{C}$ and annealing for $1 \mathrm{~min}$ at $60^{\circ} \mathrm{C}$. The spliced products of the minigenes were detected with Exon4.F and pcDNA.R primers. The PCR products were loaded onto $1 \%$ agarose gel and run at $100 \mathrm{~V}$ for $30 \mathrm{~min}$. following EtBr staining, the DNA bands were cut and dissolved using a Gel Extraction kit (Cosmo Genetech Co., Ltd.), then sent for sequencing to confirm the identity of the bands. All of the primer sequences are listed in Table I.

\section{Results}

PTCs in exon 5 do not cause degradation of all spliced and partially spliced isoforms from RON pre-mRNA. To observe the effects of PTCs on RON pre-mRNA splicing, a minigene was constructed, in which a TGA stop-codon was produced through a T-A point mutation, located 51 nucleotides (nt) downstream from the $3^{\prime}$ splice-site of exon 5 (E5/SC-1; Fig. 1A). RT-PCR analysis of the wild type (WT) minigene revealed that the following isoforms were produced: An isoform in which
Table I. Primers used for PCR and RT-PCR.

\begin{tabular}{ll}
\hline Name & \multicolumn{1}{c}{ Sequence, 5'-3' } \\
\hline Construct & \\
E4Bam.F & GTTAGGGATCCGTTTTCCAGGTACCTATC \\
& CAAG \\
E7Xho.R & ATTACCTCGAGCGTGCTAGCAGACACTCA \\
& GTC \\
E5-M1.F & CTGACCCTGTGAGGCTCCAACTTC \\
E5-M1.R & GAAGTTGGAGCCTCACAGGGTCAG \\
E5-M2.F & TCTGGTGCCTTAGGGAACCC \\
E5-M2.R & GGGTTCCCTAAGGCACCAGA \\
E6Tins.F & GAGGCTTCTCTTTTCATGGTG \\
E6Tins.R & CACCATGAAAAGAGAAGCCTC \\
E7Gdel.F & TCTTAGGAGCCATGCTGATAG \\
E7Gdel.R & TATCAGCATGGCTCCTAAGAG \\
E6Ains.F & CCACCGGGCAAAGCACTTCC \\
E6Ains.R & GGAAGTGCTTTGCCCGGTGG \\
E7Tdel.F & CAACCCCTCTTGGCCCACGG \\
E7Tdel.R & CCGTGGGCCAAGAGGGGTTG
\end{tabular}

\section{RT-PCR}

$\begin{array}{ll}\text { Exon4.F } & \text { GTTTTCCAGGTACCTATCCAAG } \\ \text { pcDNA.R } & \text { TCCACCACCCTGTTGCTGTA }\end{array}$

PCR, polymerase chain reaction; RT, reverse transcription; F, forward; R, reverse.

intron 4 is spliced but other introns are not spliced (I4/ex); an isoform in which intron 4 and intron 6 are spliced but intron 5 is not spliced (I4I6/ex); an isoform in which cryptic spliced site in intron 5 activated (I5/p/ex) (24); an isoform in which both exon 5 and 6 are included (E5E6/in); and an isoform in which exon 5 is included but exon 6 is excluded (E5/in; lane 2; Fig. 1B). The results of the current study were consistent with the prior hypothesis that PTCs in exons induce and regulate the NMD signaling pathway. RT-PCR analysis of the E5/SC-1 minigene revealed that I4/ex was markedly decreased and the I5/p/ex, E5E6/in and E5/in isoforms were almost absent. However, the expression of the I4I6/ex isoform from this mutated minigene was not observed to differ from that of the WT product. The results of the current study indicate that, although most isoforms were reduced significantly by PTCs on exon 5, a partially spliced isoform still survived in the mutant minigene.

Different PTC locations in exon 5 have various effects on the NMD of the spliced and partially spliced isoforms of RON pre-mRNA. It was hypothesized that changing the location of the PTCs in RON exon 5 may affect the alternative splicing of exons 5 and 6 in RON pre-mRNA. A second mutant minigene was constructed, in which a stop-codon was produced $91 \mathrm{nt}$ downstream from the 3' splice-site of exon 5, through a G-T point mutation (E5/SC-2; Fig. 1A). It was observed that the majority of the exon isoforms, including I4/ex, I4/I6/ex, I5/p/ex and E5E6/in, were markedly reduced in the E5/SC-2 minigene (Fig. 1B; lane 4); however, the E5/in isoform was not 
A

wt

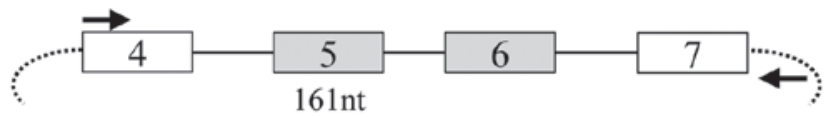

E5/SC-1

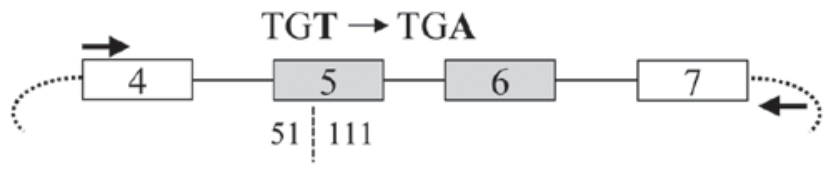

$161 \mathrm{nt}$

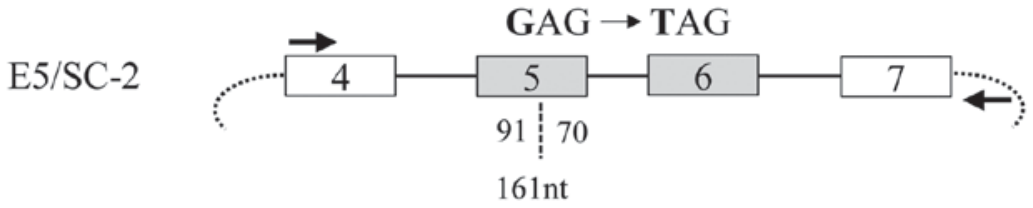

B

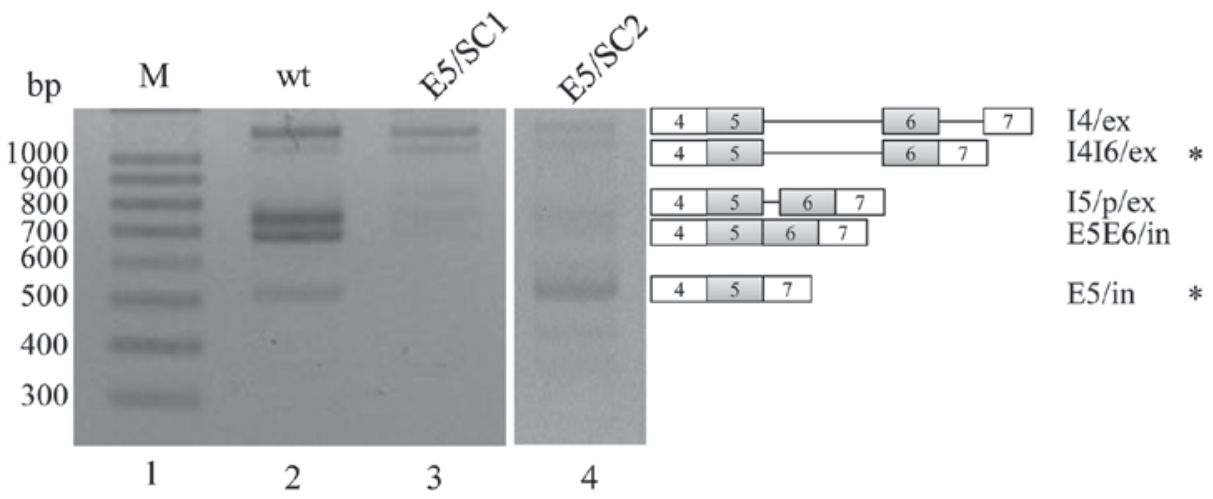

Figure 1. Various locations of the stop-codon in exon 5 have various effects on the degradation of the spliced and partially spliced isoforms from RON pre-mRNA. (A) The construction of the E5/SC-1 (T-to-A substitution 51 nucleotides downstream from the 3' splice-site of exon 5) and E5/SC-2 (G-to-T substitution 91 nucleotides downstream from the $3^{\prime}$ splice-site of exon 5) minigenes is shown. Exons are indicated with boxes; introns are marked with lines; vector sequences are presented with arcs. The locations of the stop-codons are shown with the numbers of nucleotides. Primers used for RT-PCR analysis are indicated with arrows. (B) Results of the RT-PCR analysis of E5/SC-1 and E5/SC-2 minigenes are presented. The identities of each of the isoforms are indicated at the right panel. The isoforms observed to not decrease are designated with asterisks. RON, récepteur d'origine nantais; RT-PCR, reverse transcription-polymerase chain reaction; wt, wild type; M, molecular weight ladder; I4/ex, intron 4 is spliced; I4I6/ex, introns 4 and 6 are spliced; I5/p/ex, cryptic splice site in intron 5 is activated; E5E6/in, exons 5 and 6 are included; E5/in, exon 5 included and exon 6 excluded.

reduced in this mutant. Thus, the E5/in isoform was resistant to degradation by the PTC-mediated NMD signaling pathway. The results (Fig. 1B) indicate that, although PTCs on exon 5 induced a marked decrease in the majority of the spliced or partially spliced mRNA isoforms, specific isoforms may still survive the PTC-induced NMD. However, the identities of the surviving isoforms are dependent on the location of the PTCs in the RON pre-mRNA.

PTCs produced by inserting T or A nts do not induce NMD of specific isoforms. The current study investigated whether the PTCs produced by inserting a T residue in exon 6 may have similar effects on RON pre-mRNA splicing. To ensure that the spliced product was still capable of encoding the protein, a $\mathrm{T}$ residue was also deleted from exon 7. In the E6-7/SC-1 minigene, the inserted $\mathrm{T}$ was located 7-nt upstream from the 5 ' splice site of exon 6 , and the deleted $G$ residue was located 7-nt downstream from the 3 ' splice site of exon 7 (Fig. 2A). The RT-PCR results of the E6-7/SC-1 minigene were consistent with the PTC-mediated NMD hypothesis, and demonstrated that the majority of spliced or partially spliced isoforms, including I4/ex, I4I6/ex, I5/p/ex and E5E6/in, were markedly decreased compared with the WT minigene. However, the production of the isoform wherein exon 5 was included but exon 6 was excluded (E5/in) was markedly increased. Furthermore, the isoform in which both exons 5 and 6 were excluded (E5E6/ex) was markedly increased; E5E6/ex was not produced from the WT minigene. Thus, E5/in and E5E6/ex were observed to survive the NMD signaling pathway.

The present study further investigated whether the effects of inserted PTCs were dependent on their respective location. An A residue was inserted 64-nt upstream from the 5 ' splice-site of exon 6 , and a $\mathrm{T}$ residue was deleted 66-nt downstream from the 3 ' splice-site of exon 7 (E6-7/SC-2). The results demonstrated that, although I4/ex, I4I6/ex, I5/p/ex and E5E6/in isoform production from the E6-7/SC-2 minigene was markedly decreased, E5/in isoform was also significantly increased in the E6-7/SC-1 and E6-7/SC-2 minigenes. In addition, the E5E6/ex isoform was more markedly increased in the E6-7/SC-2 minigene than in the E6-7/SC1 minigene 
$\mathbf{A}$

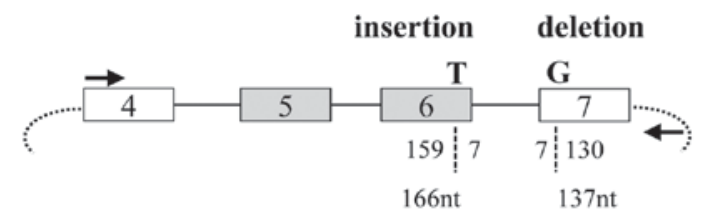

E6-7/SC-2
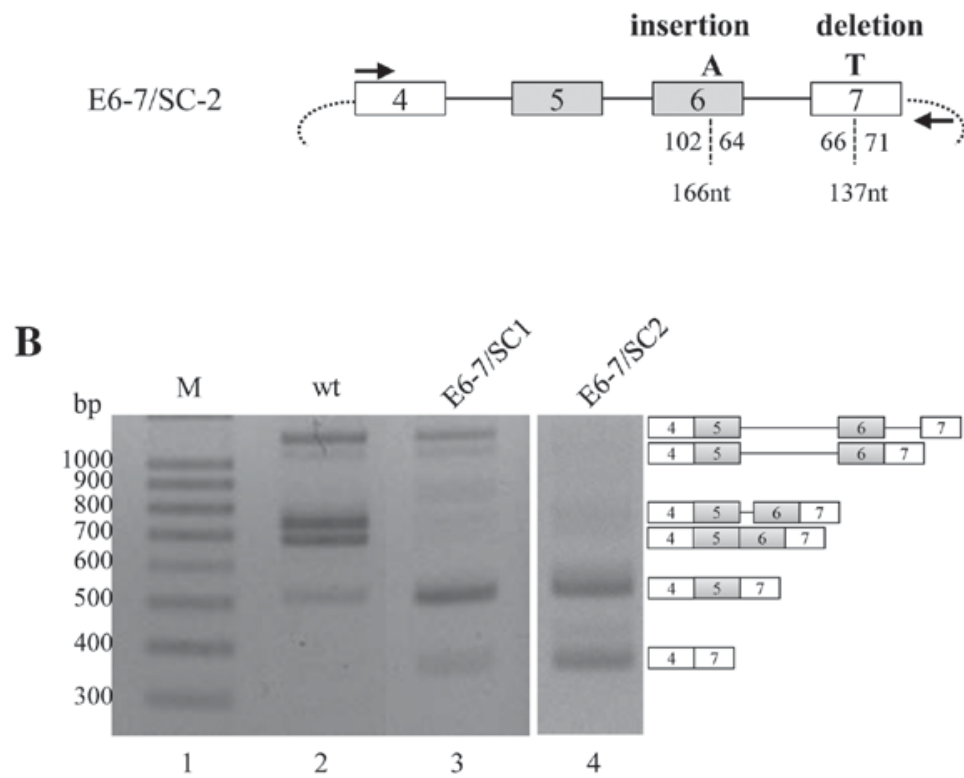

I4/ex

I4I6/ex

I5/p/ex

E5/in *

E5E6/ex *

Figure 2. Stop-codons produced by inserting T or A nucleotides do not induce nonsense-mediated mRNA decay of specific isoforms. (A) The construction of the E6-7/SC-1 (T insertion on exon 6 and G deletion on exon 7) and E6-7/SC-2 (An insertion on exon 6 and T deletion on exon 7) minigenes is exhibited. The locations of the inserted and deleted nucleotides are indicated. Primers used in the RT-PCR analysis are marked with arrows. (B) RT-PCR analysis of the E6-7/SC-1 and E6-7/SC-2 minigenes is presented. The identities of each of the isoforms produced are indicated at the right panel. The isoforms increased in the mutant minigenes are designated with asterisks. RT-PCR, reverse transcription-polymerase chain reaction; wt, wild type; M, molecular weight ladder; I4/ex, intron 4 is spliced; I4I6/ex, introns 4 and 6 are spliced; I5/p/ex, cryptic splice site in intron 5 is activated; E5E6/in, exons 5 and 6 are included; E5/in, exon 5 included and exon 6 excluded; E5E6/ex, exons 5 and 6 are excluded.

(Fig. 2B). In conclusion, the results demonstrated that PTCs created through both the insertion and the deletion of certain nts may induce a marked decrease of the majority of spliced or unspliced isoforms, but also stimulate the production of specific isoforms, of RON pre-mRNA.

\section{Discussion}

It has been demonstrated in a previous study that mutations in minigenes can affect the splicing of alternative exons (12). The results of the present study confirmed that the differential location of PTCs on alternative exons 5 and 6 may induce NMD of the majority of the spliced or partially spliced exon isoforms. A minority of RON alternative-splicing products, exon 6 excluded (E5/in), exon 5 and 6 excluded (E5E6/ex) and intron 5 unspliced isoforms, were observed to have survived the NMD pathway. Furthermore, the varieties of these isoforms are dependent on the location of PTCs in the mRNA. Therefore, the current study provided an insight into the regulation of NMD in alternative splicing.

The present study investigated the effect of PTC-mediated NMD on the alternative splicing of the RON proto-oncogene. The results of the current study were consistent with those of previous reports, demonstrating that PTCs, created by the substitution or insertion of nts, induce NMD of the majority of spliced or unspliced isoforms, as indicated by the significantly decreased production of these isoforms. However, by contrast with previous hypotheses (25), specific isoforms of alternatively spliced RON pre-mRNA were not reduced or increased as a consequence of the mutations. The isoform identities varied in the mutant minigenes that harbored PTCs at alternative locations on exons 5 or 6 , and were created using different approaches. It was observed that the isoform that includes exon 5 but not exon 6 (E5/in) was increased in the majority of stop-codon-including minigenes; thus the E5/in isoform may have included an RNA sequence that was resistant to degradation by the NMD signaling pathway. The results of the current study also demonstrated that E5E6/ex was increased in the presence of certain PTCs. The underlying mechanisms that may allow these isoforms to resist the NMD signaling pathway must be determined through further studies. It is possible that these isoforms possess specific sequences that facilitate the recruitment of anti-NMD signaling pathway proteins, and also include the sequences that promote alternative splicing.

Although it was previously reported that PTCs cause degradation of mRNA through NMD, the results of the present study indicated that PTCs located at different positions may induce the survival of various spliced or unspliced isoforms. Thus, in addition to inducing NMD, PTCs have other important roles in the survival of spliced, unspliced and partially spliced isoforms. The results suggest that the insertion of PTCs at specific positions by gene editing may potentially enhance 
the survival of the isoforms that encode tumor suppressors or metastasis suppressors. Similarly, the insertion of PTCs at other specific positions could cause degradation of the isoforms that produce oncogenes or tumor metastasis genes.

\section{Acknowledgements}

This study was supported by the NRF-2015R1A2A1A1505 4247 grant to Haihong Shen, the NRF-2016R1A2B1007135 grant to Xuexiu Zheng and Cell Logistics Research Center (grant no. 2016R1A5A1007318) funded by the National Research Foundation (NRF) of Korea, and an integrative aging research grant at the Gwangju Institute of Science and Technology (GIST).

\section{References}

1. Ronsin C, Muscatelli F, Mattei MG and Breathnach R: A novel putative receptor protein tyrosine kinase of the met family. Oncogene 8: 1195-1202,1993.

2. Zhao S, Ammanamanchi S, Brattain M, Cao L, Thangasamy A, Wang J and Freeman JW: Smad4-dependent TGF-beta signaling suppresses RON receptor tyrosine kinase-dependent motility and invasion of pancreatic cancer cells. J Biol Chem 283: 11293-11301, 2008.

3. Thangasamy A, Rogge J and Ammanamanchi S: Recepteur d'origine nantais tyrosine kinase is a direct target of hypoxia-inducible factor-1alpha-mediated invasion of breast carcinoma cells. J Biol Chem 284: 14001-14010, 2009.

4. Logan-Collins J, Thomas RM, Yu P, Jaquish D, Mose E, French R, Stuart W, McClaine R, Aronow B, Hoffman RM, et al: Silencing of RON receptor signaling promotes apoptosis and gemcitabine sensitivity in pancreatic cancers. Cancer Res 70: 1130-1140, 2010.

5. Iwama A, Okano K, Sudo T, Matsuda Y and Suda T: Molecular cloning of a novel receptor tyrosine kinase gene, STK, derived from enriched hematopoietic stem cells. Blood 83: 3160-3169, 1994.

6. Yoshimura T, Yuhki N, Wang MH,Skeel A and Leonard EJ: Cloning, sequencing, and expression of human macrophage stimulating protein (MSP, MST1) confirms MSP as a member of the family of kringle proteins and locates the MSP gene on chromosome 3.J Biol Chem 268: 15461-15468, 1993.

7. Wang MH, Ronsin C, Gesnel MC, Coupey L, Skeel A, Leonard EJ and Breathnach R: Identification of the ron gene product as the receptor for the human macrophage stimulating protein. Science 266: 117-119, 1994.

8. Lu Y, Yao HP and Wang MH: Multiple variants of the RON receptor tyrosine kinase: Biochemical properties, tumorigenic activities, and potential drug targets. Cancer Lett 257: 157-164, 2007.

9. Collesi C, Santoro MM, Gaudino G and Comoglio PM: A splicing variant of the RON transcript induces constitutive tyrosine kinase activity and an invasive phenotype. Mol Cell Biol 16: 5518-5526, 1996.
10. Ghigna C, Giordano S, Shen H, Benvenuto F, Castiglioni F, Comoglio PM, Green MR, Riva S and Biamonti G: Cell motility is controlled by SF2/ASF through alternative splicing of the Ron protooncogene. Mol Cell 20: 881-890, 2005.

11. Bonomi S, di Matteo A, Buratti E, Cabianca DS, Baralle FE, Ghigna $\mathrm{C}$ and Biamonti G: HnRNP A1 controls a splicing regulatory circuit promoting mesenchymal-to-epithelial transition. Nucleic Acids Res 41: 8665-8679, 2013.

12. Moon H, Cho S, Loh TJ, Oh HK, Jang HN, Zhou J, Kwon YS, Liao DJ, Jun Y, Eom S, et al: SRSF2 promotes splicing and transcription of exon 11 included isoform in Ron proto-oncogene. Biochim Biophys Acta 1839: 1132-1140, 2014.

13. Zhou YQ, He C, Chen YQ, Wang D and Wang MH: Altered expression of the RON receptor tyrosine kinase in primary human colorectal adenocarcinomas: Generation of different splicing RON variants and their oncogenic potential. Oncogene 22: 186-197, 2003.

14. Wang MH, Kurtz AL and Chen Y: Identification of a novel splicing product of the RON receptor tyrosine kinase in human colorectal carcinoma cells. Carcinogenesis 21: 1507-1512, 2000.

15. Chen YQ, Zhou YQ, Angeloni D, Kurtz AL, Qiang XZ and Wang MH: Overexpression and activation of the RON receptor tyrosine kinase in a panel of human colorectal carcinoma cell lines. Exp Cell Res 261: 229-238, 2000.

16. Green MR: Pre-mRNA splicing. Annu Rev Genet 20: 671-708, 1986.

17. Black DL: Mechanisms of alternative pre-messenger RNA splicing. Annu Rev Biochem 72: 291-336, 2003.

18. Berglund JA, Chua K, Abovich N, Reed R and Rosbash M: The splicing factor BBP interacts specifically with the pre-mRNA branchpoint sequence UACUAAC. Cell 89: 781-787, 1997.

19. Nelson KK and Green MR: Mammalian U2 snRNP has a sequence-specific RNA-binding activity. Genes Dev 3: 1562-1571, 1989.

20. Isken $\mathrm{O}$ and Maquat LE: The multiple lives of NMD factors: Balancing roles in gene and genome regulation. Nat Rev Genet 9: 699-712, 2008.

21. Chang YF,Imam JS and Wilkinson MF: The nonsense-mediated decay RNA surveillance pathway. Annu Rev Biochem 76: 51-74, 2007.

22. Valacca C, Bonomi S, Buratti E, Pedrotti S, Baralle FE, Sette C, Ghigna C and Biamonti G: Sam68 regulates EMT through alternative splicing-activated nonsense-mediated mRNA decay of the SF2/ASF proto-oncogene. J Cell Biol 191: 87-99, 2010

23. Saltzman AL, Kim YK, Pan Q, Fagnani MM, Maquat LE and Blencowe BJ: Regulation of multiple core spliceosomal proteins by alternative splicing-coupled nonsense-mediated mRNA decay. Mol Cell Biol 28: 4320-4330, 2008

24. Ma Q, Zhang K, Yao HP, Zhou YQ, Padhye S and Wang MH: Inhibition of MSP-RON signaling pathway in cancer cells by a novel soluble form of RON comprising the entire sema sequence. Int J Oncol 36: 1551-1561, 2010.

25. Gong Q, Zhang L, Vincent GM, Horne BD and Zhou Z: Nonsense mutations in hERG cause a decrease in mutant mRNA transcripts by nonsense-mediated mRNA decay in human long-QT syndrome. Circulation 116: 17-24, 2007. 